\title{
Thoracic Kyphotic Deformity Secondary to Old Pseudomonas aeruginosa Spondylodiscitis in an Immunocompromised Patient With Persistent Infection Foci-A Case Report
}

\author{
ANOUAR BOURGHLI, MD, ${ }^{1}$ LOUIS BOISSIERE, $\mathrm{MD},{ }^{2}$ IBRAHIM OBEID, $\mathrm{MD}^{2}$ \\ ${ }^{I}$ Orthopedic and Spinal Surgery Department, Kingdom Hospital, Riyadh, Saudi Arabia, ${ }^{2}$ Orthopedic Spinal Surgery Unit 1, Bordeaux Pellegrin Hospital, \\ Bordeaux, France
}

\begin{abstract}
Background: Kyphosis secondary to pyogenic spondylodiscitis is rare and its management can be very challenging.

Methods: In this report, we present the case of a 28-year-old woman, with past history of type 1 diabetes and kidney failure on hemodialysis. Her current complaint is chronic middle and low back pain with kyphotic attitude. She had undergone posterior fixation for T12 fracture 3 years earlier, which was complicated by surgical site infection to Pseudomonas aeruginosa, with secondary kyphosis proximally. X-ray showed a $64^{\circ}$ kyphosis with complete fusion between T8 and T10, and MRI showed persistent infection foci.

Results: The patient underwent a pedicle subtraction osteotomy at the level of $\mathrm{T} 9$ with instrumentation from $\mathrm{T} 5$ to L1. Thoracic kyphosis was corrected to $39^{\circ}$. Samples taken from the remaining collections returned positive for multidrug-resistant Pseudomonas aeruginosa, and the patient was kept on intravenous antibiotic (Colistine) for 2 months. She could walk on day 1, with a satisfactory clinical and radiological result at 3 years.

Conclusions: Literature is sparse on the management of post-pyogenic infection kyphosis in immunocompromised patients. The current case shows that aggressive correction techniques such as pedicle subtraction osteotomy can be performed in such cases but within a multidisciplinary team to deal simultaneously with the different issues of the fragile patient.
\end{abstract}

\section{Complications}

Keywords: kyphosis, pyogenic spondylodiscitis, Pseudomonas aeruginosa, pedicle subtraction osteotomy, immunocompromised patient

\section{INTRODUCTION}

The number of pyogenic spinal infections has increased during the past decades in relation to the greater number of older patients and also immunocompromised patients (chronic renal failure, diabetes type 1, long-term steroid intake, HIV, chronic immunosuppression). ${ }^{1}$ The spine accounts for about $0.15 \%$ to $3.90 \%$ of all cases of osteomyelitis, but despite this relatively low rate, its consequences may be devastating, resulting in severe back pain, kyphotic deformity, and neurological impairment. ${ }^{2}$ Secondary kyphosis after infection has been widely described in the literature for tuberculosis cases $^{3,4}$ but rarely for pyogenic microorganisms. ${ }^{5,6}$ Staphylococcus aureus is the most common organism identified with postoper- ative spinal infections, followed by Streptococcus. Pseudomonas aeruginosa is a relatively uncommon cause, as it accounts for about $5 \%$ of the cases. ${ }^{7,8}$ In a recent study, ${ }^{9}$ it was stated that occult infections are present in $9.3 \%$ of patients who underwent revision spine surgery and hardware removal, although they did not have clinical signs of infection; however, the common inflammatory markers such as $\mathrm{C}$ reactive protein, erythrocyte sedimentation rate, and procalcitonin do not seem sensitive enough to detect these occult infections. The combination of a rigid kyphosis secondary to a previous postoperative spinal infection and an occult infection remaining from that postoperative infection is not common and may require a specific management, especially when aggressive surgery is 


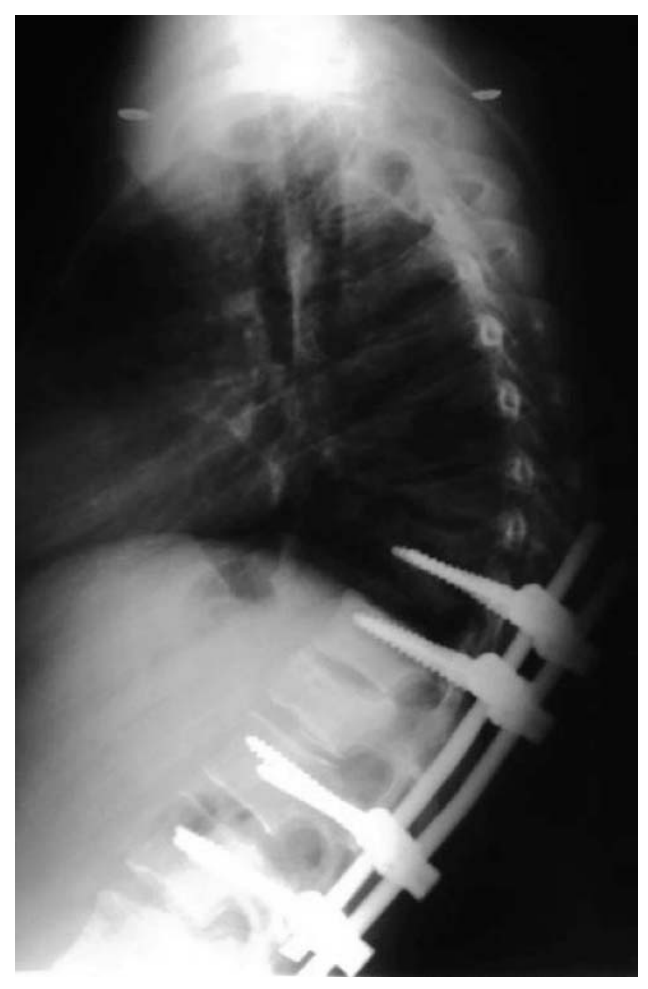

Figure 1. Thoraco-lumbar sagittal $\mathrm{X}$-ray 3 years before presentation showing the onset of the kyphosis proximally to the initial construct with the T10 screws inside the T9-10 disk space.

required and the patient is in the "immunocompromised" category.

\section{CASE DESCRIPTION}

A 28-year-old woman had been complaining of middle and low back pain with kyphotic attitude for almost 3 years. Her past history revealed insulindependent type 1 diabetes with kidney failure requiring 3 hemodialysis sessions per week; she had sustained 3 years before, in another country, a T12 compression fracture that was surgically treated with T10 to L2 posterior fixation; this was complicated by surgical-site infection to multidrugresistant $P$. aeruginosa with spondylodiscitis T8-9 and T9-10. According to the patient, she was managed conservatively with intravenous (IV) antibiotics for over 6 weeks, but the discharge would not stop and the wound did not heal. Hardware was removed at 3 months, which led to progressive healing but with the occurrence of a secondary kyphosis proximally. Total period of IV antibiotics was around 6 months.

Physical examination revealed middle back pain at the level of the previous surgery with a significant

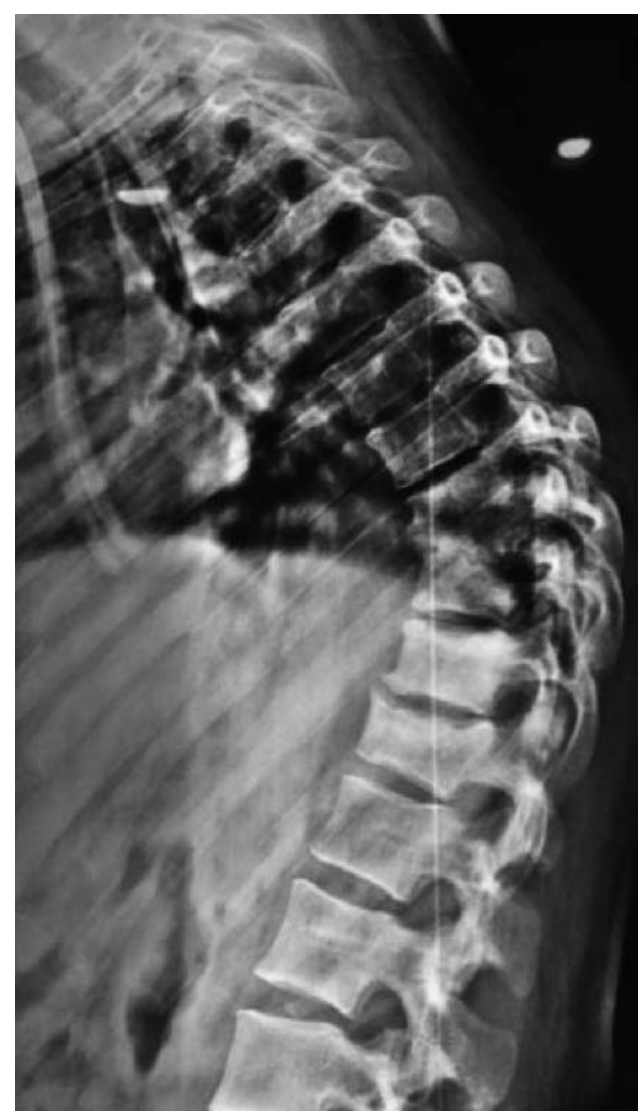

Figure 2. X-ray showing a significant kyphosis 6 months after hardware removal.

thoracic kyphosis clinically, associated with low back pain probably due to a facet joint syndrome because hyperlordosis could be noted. A 3-year-old thoracic x-ray brought by the patient showed the onset of the kyphosis proximally to the initial construct with the T10 screws inside the T9-10 disk space (Figure 1), and according to the patient, the rods at that time were palpable below the skin proximally but not out of the skin. Six months after hardware removal, thoracic x-ray revealed a significant kyphosis (Figure 2). Full-spine anteroposterior and lateral $\mathrm{x}$-rays at the time of presentation revealed a thoracic kyphosis of $64^{\circ}$, with compensatory hyperlordosis of $67^{\circ}$ and no coronal deformity (Figure 3a, b). Computed tomography (CT) scan confirmed the complete fusion between $\mathrm{T} 8$ and T10, with vertebral body sclerosis between T8 and T12 (Figure 4). A potential differential diagnosis is stable nonunion, but different coronal and sagittal images showed no differentiation between the T8 and T9 vertebral bodies laterally, thus confirming the fusion. MRI showed paraspinal collections (anterior and lateral) suggestive of remaining 

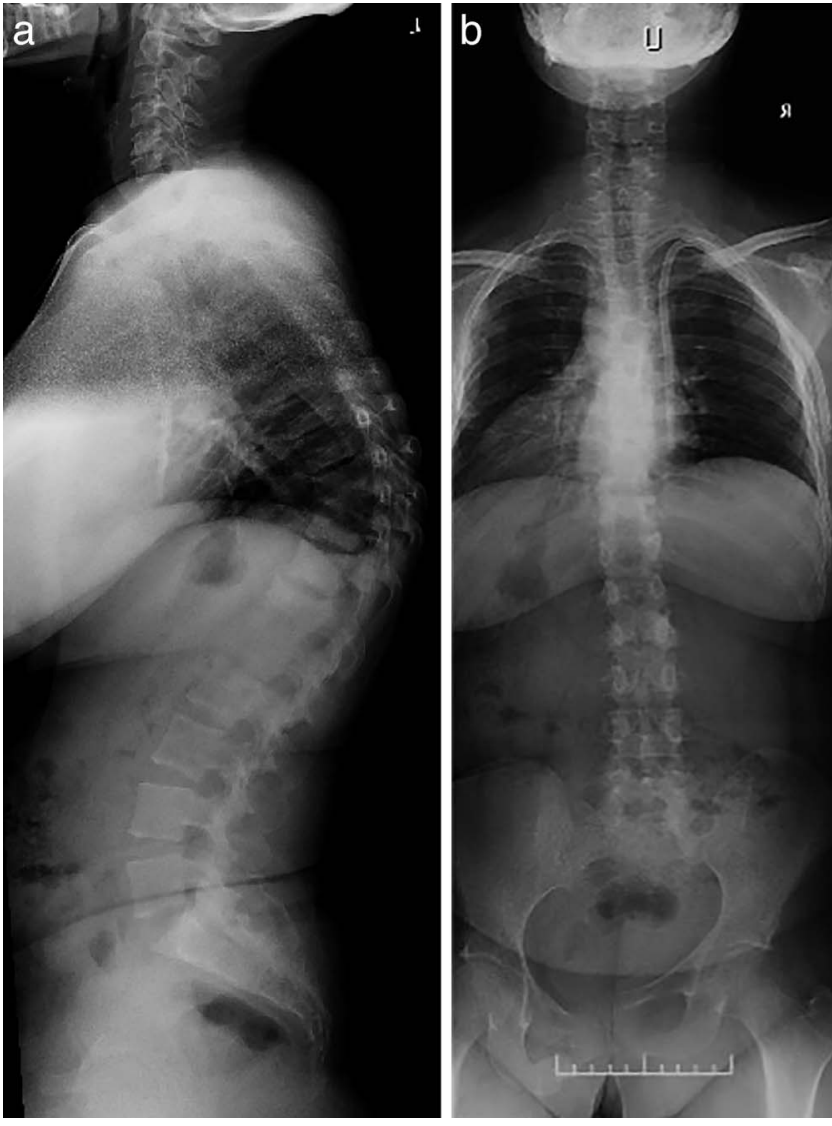

Figure 3. Full spine anteroposterior and lateral $x$-rays at the time of presentation revealing a thoracic kyphosis of $64^{\circ}$, with compensatory hyperlordosis of $67^{\circ}$ and no coronal deformity ( $a$ and $b$ ).

infection foci, in addition to spinal canal narrowing at the kyphosis level (Figure 5a, b).

After discussion with the anesthesiologist, nephrologist, and endocrinologist, the patient underwent a pedicle subtraction osteotomy (PSO) at the level of T9 with instrumentation from T5 to L1.

\section{SURGICAL TECHNIQUE}

The patient was installed in a prone position, on 4 cushions (Figure 6a). During the surgery, we used transcranial motor-evoked potentials, somatosensory evoked potentials, and free-running electromyography (EMG) of the lower extremities as well as evoked EMGs with pedicle-screw stimulation.

The operative field was exposed from T5 to L1; a posterior cutaneous midline incision was made. The spine was exposed subperiosteally, going laterally to the costotransverse junction. Resection of the inferior articular processes at all levels was performed bilaterally to provide maximum flexibility to the spine. We next used the freehand technique to

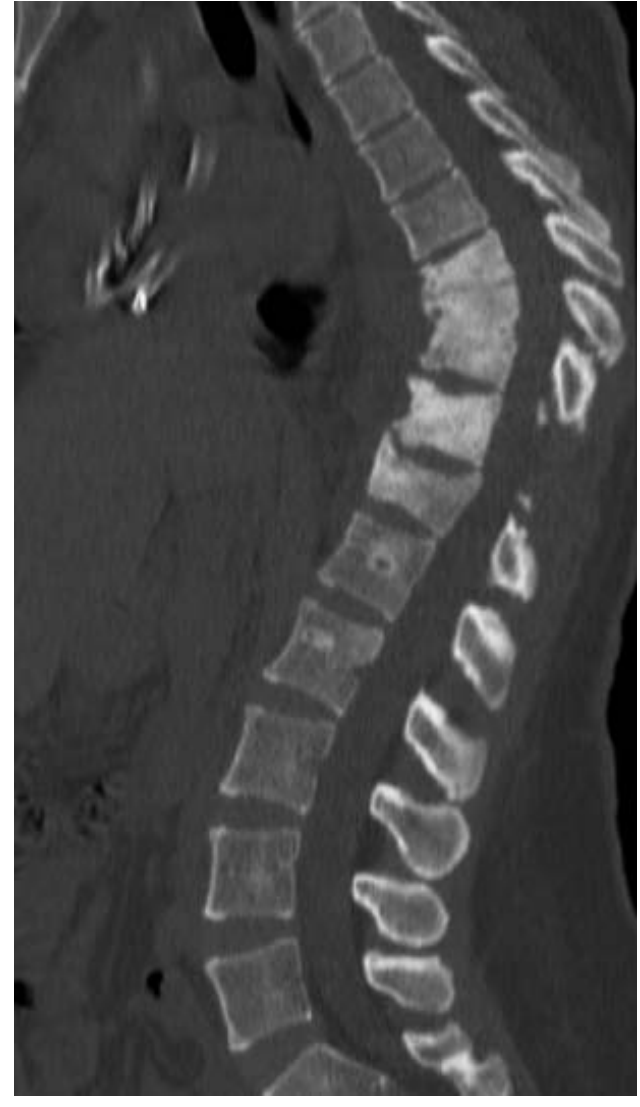

Figure 4. Computed tomography scan showing the complete fusion between $\mathrm{T} 8$ and T10, with vertebral body sclerosis between T8 and T12.

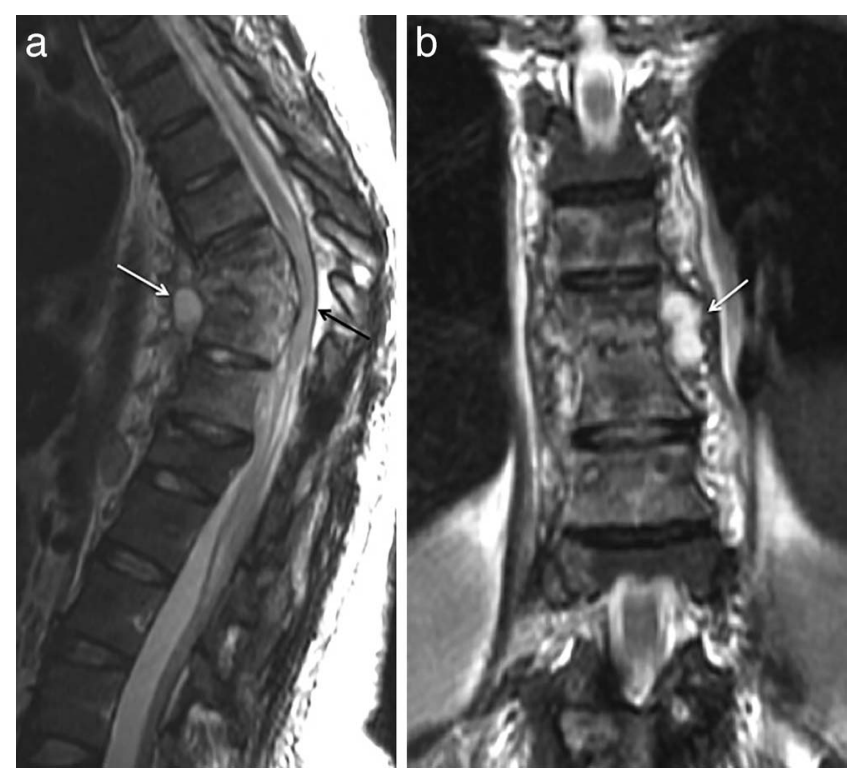

Figure 5. MRI revealing paraspinal collections anteriorly (a, white arrow) and laterally ( $b$, white arrow) suspicious of remaining infection foci, in addition to spinal canal narrowing at the kyphosis level (a, black arrow). 

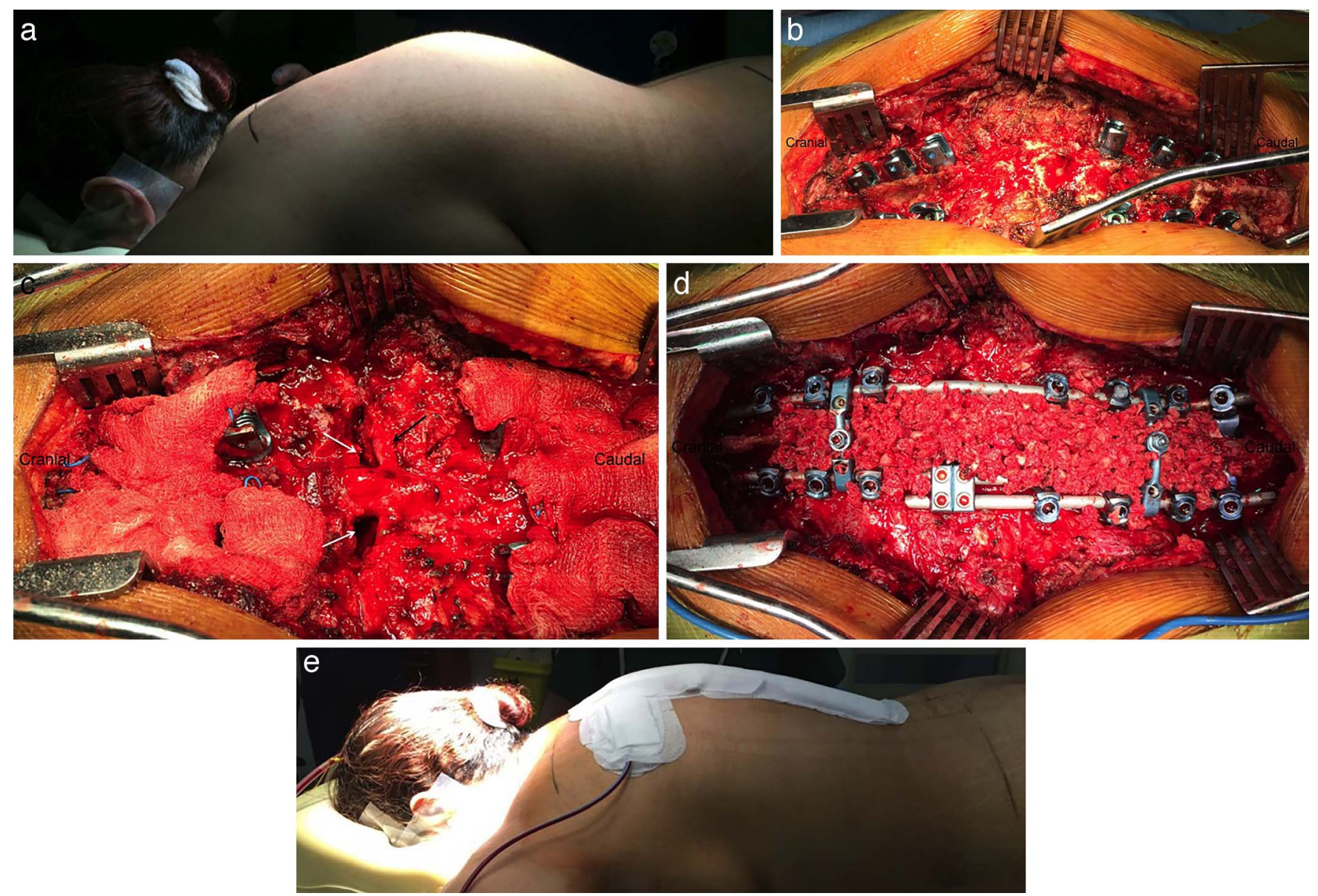

Figure 6. Intraoperative images showing the thoracic hump (a), spinal exposure, and screws insertion from T5 to L1 but sparing T8 to T10 (b); pedicle subtraction osteotomy performed at the level of T9 (c, white arrows); the right T9 root is clearly visible (c, black arrow); reduction of the kyphosis and placement of autologous bone graft (d); clinical aspect at the end of the procedure (e).

place the pedicle screws from T5 to T7 and from T10 to L1 (Figure 6b); 2 sublaminar offset hooks were put at the distal level of the construct to increase the resistance to pullout forces. To expose the lateral wall of T9, the transverse processes were removed with a rongeur, and the proximal $3 \mathrm{~cm}$ of the right and left ribs (including the rib head) were removed. A Cobb elevator was then placed, bilaterally, on the lateral wall of the vertebra and moved anteriorly to the anterolateral quadrant to retract all the lateral soft tissues. A suspicious collection was noted on the left side and several samples were taken and sent for culture; a complete Surgicel (Johnson \& Johnson, New Brunswick, New Jersey) was left in place to maintain a safe distance between the bone and the soft tissues. Two complete foraminotomies (both cephalad and caudad to the pedicles on both sides) were made, achieved by complete laminectomy of the concerned level, with partial laminectomies of the levels just above and just below. This enabled surrounding of the pedicles. Both pedicles were then removed, exposing the posterior wall of the vertebra, and the nerve roots above and below were identified. After retraction of the lower nerve root, 2 osteotomes are placed above and below each pedicle. Cancellous bone was removed in a wedge fashion from posterior to anterior on both sides. Because retraction of the cord is prohibited at the level of the thoracic spine, direction of the osteotomes for removal of the medial part of the bone becomes horizontal, and the medial part of the posterior wall was finally removed with an up-angled pituitary rongeur (Figure 6c). For the correction technique, at first, cantilevering of the spine was performed with 2 prebent titanium alloy rods connected by a domino on the left side. Further closure of the osteotomy by compression with the use of the domino was applied; the rod was then completely locked. During the osteotomy closure, an audible crack was noted; 


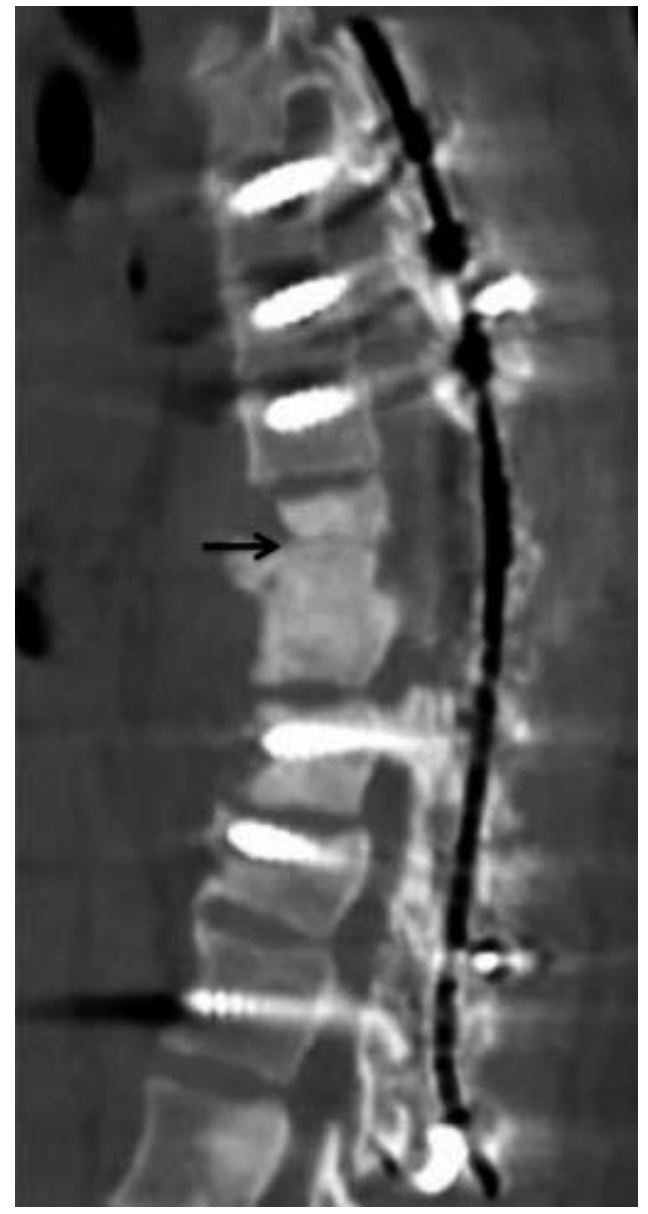

Figure 7. Postoperative CT scan confirming the kyphosis reduction with boneon-bone contact at the osteotomy level.

this was due to the fracture of an anterior osteophyte and the complete sclerosis at the osteotomy site. The bone-on-bone contact at the osteotomized level was checked, and the spinal cord was carefully controlled because kinking could occur in case of important reduction. The contralateral rod was placed and secured. The fixation was completed by the placement of two cross-link connectors between the 2 rods, 1 proximal and 1 distal. The prepared autologous bone grafts were placed to cover the maximum surface (Figure 6d). Postoperative improvement of the gibbosity could be seen (Figure 6e).

\section{RESULTS}

The patient could walk on day 2 with a thoracic lumbar orthosis, to be used for 3 months, and with assistance from a physical therapist. She was discharged on day 14 with proper wound healing. Postoperative CT scan confirmed good correction at
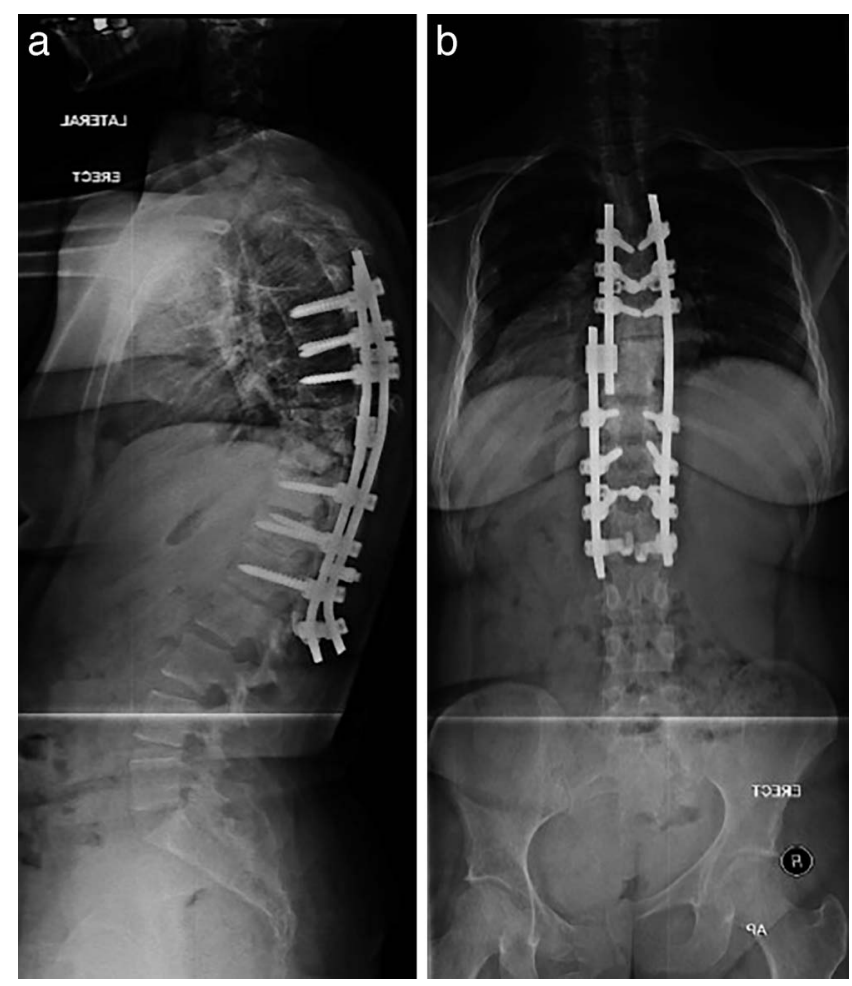

Figure 8. Full-spine $x$-rays 3 years after the surgery showing a stable reduction and construct.

the PSO level with a bone-on-bone contact (Figure 7 ), and x-rays showed a correction of the thoracic kyphosis to $39^{\circ}$ with correction of the compensatory hyperlordosis to $50^{\circ}$. Samples taken from the remaining collections returned positive for multidrug-resistant $P$. aeruginosa sensitive to only Colistine, and the patient was kept on intravenous antibiotic for 2 months. She showed satisfactory clinical and radiological result at 3 years (Figure 8a, b).

\section{DISCUSSION}

Thoracic kyphosis secondary to pyogenic spondylodiscitis is challenging when it comes to its surgical management, especially in a patient with comorbidities. In fact, spinal surgery is associated with significantly higher mortality rates in patients with chronic kidney disease compared with patients with normal kidney function. ${ }^{10}$ Therefore, management should be done within a multidisciplinary team with permanent involvement of different specialties; thus, in addition to the spine surgeon and infection disease specialist, an endocrinologist and nephrologist must be involved if diabetes and renal failure are present; the sugar rate must be 
closely monitored and well controlled prior to the surgery, ${ }^{11}$ and dialysis sessions must be well scheduled around the surgical date, with usually 1 session the day before. ${ }^{12-14}$

There is a controversy in the surgical management of secondary kyphosis after pyogenic infection because no evidence-based guidelines exist, given that what is mainly found in the literature is case reports. Surgery can be achieved by an anterior-only approach with instrumentation ${ }^{5}$ when infectious involvement has been recent (less than 6 weeks) with no rigid deformity, which enables correction and reconstruction with multilevel cages in the apical disks with anterior instrumentation. Surgery can also be performed by a double approach that can be either anterior/posterior or posterior/anterior in the same setting or sequentially ${ }^{15}$; the anterior part enables debridement, decompression, and anterior fusion with a strut graft, and the posterior part enables posterior instrumentation and fusion. This technique is also advised in cases of kyphosis secondary to a recent infection with an ongoing infectious process with hypersignal seen at the disk levels on the MRI, because it provides a smaller loss of correction when compared with either the posterior-only or anterior-only approach. To palliate the loss of correction of the posterior-only technique, a technique of long posterior instrumentation with short fusion and without debridement of the infected tissue has been described ${ }^{16}$ that can strongly reduce the kyphotic deformity and prevent loss of reduction. But in cases of a rigid kyphosis secondary to an old infection with complete vertebral fusion, it is advised to perform a posterior-only approach with the use of aggressive corrective techniques such as PSO or vertebral column resection. This has been widely described in the literature for tuberculosis patients, ${ }^{17,18}$ as it allows an important amount of correction in a onestage surgery.

$P$. aeruginosa is a relatively uncommon cause of pyogenic spondylodiscitis, as it accounts for only $5 \%$ of the cases, ${ }^{7,8}$ mainly encountered in the background of IV drug use and immunocompromised patients. It is frequently multidrug-resistant, and therefore the close involvement of the infection disease specialist is mandatory during the course of treatment.

In the case of our patient, given the angulation of the kyphosis, with complete vertebral fusion at the apex, and also the fragility of the patient with renal failure, a posterior-only approach with pedicle subtraction osteotomy at the level of T9 was done with instrumentation from T5 to L1. Such an aggressive correction technique could be successfully performed in this case, even when the initial responsible germ was multidrug-resistant $P$. aeruginosa, but within a multidisciplinary team to simultaneously deal with the different issues of the fragile patient.

\section{REFERENCES}

1. Rezai AR, Woo HH, Errico TJ, Cooper PR. Contemporary management of spinal osteomyelitis. Neurosurgery. 1999;44(5):1018-1025, discussion 1025-1026.

2. An HS, Seldomridge JA. Spinal infections: diagnostic tests and imaging studies. Clin Orthop Relat Res. 2006;444(March): 27-33. https://doi.org/10.1097/01.blo.0000203452.36522.97

3. Rawall S, Mohan K, Nene A. Posterior approach in thoracolumbar tuberculosis: a clinical and radiological review of 67 operated cases. Musculoskelet Surg. 2013;97(1):67-75. https://doi.org/10.1007/s12306-012-0235-y

4. Zeng Y, Chen ZQ, Guo ZQ, Qi Q, Li WS, Sun CG. The posterior surgical treatment of old tuberculous kyphosis [in Chinese]. Zhonghua Wai Ke Za Zhi. 2012;50(1):23-27.

5. Neumayer F, Kosmopoulos V, Schizas C. Management of a post-operative multi-resistant infectious spondylitis associated with a kyphotic deformity. Acta Orthop Belg. 2009;75(4):566570 .

6. Basu S, Sreeramalingam R. Adjacent level spondylodiscitis after anterior cervical decompression and fusion. Indian $J$ Orthop. 2012;46(3):360-363. https://doi.org/10.4103/0019-5413. 96367

7. Meher SK, Jain H, Tripathy LN, Basu S. Chronic Pseudomonas aeruginosa cervical osteomyelitis. J Craniovertebr Junction Spine. 2016;7(4):276-278. https://doi.org/10.4103/ 0974-8237.193264

8. Weinstein MA, McCabe JP, Cammisa FP Jr. Postoperative spinal wound infection: a review of 2, 391 consecutive index procedures. J Spinal Disord. 2000;13(5):422-426.

9. $\mathrm{Hu} \mathrm{X}$, Lieberman IH. Revision spine surgery in patients without clinical signs of infection: how often are there occult infections in removed hardware? Eur Spine J. 2018;27(10): 2491-2495. https://doi.org/10.1007/s00586-018-5654-5

10. Bains RS, Kardile M, Mitsunaga L, et al. Does chronic kidney disease affect the mortality rate in patients undergoing spine surgery? J Clin Neurosci. 2017;43:208-213. https://doi. org/10.1016/j.jocn.2017.05.014

11. Shin JI, Phan K, Kothari P, Kim JS, Guzman JZ, Cho SK. Impact of glycemic control on morbidity and mortality in adult idiopathic scoliosis patients undergoing spinal fusion. Clin Spine Surg. 2017;30(7):E974-E980. https://doi.org/10.1097/ BSD.0000000000000447

12. Chen LH, Fu TS, Kao YH, et al. Surgical treatment of infectious spondylitis in patients undergoing hemodialysis therapy. Eur Spine J. 2010;19(12):2223-2228. https://doi.org/ 10.1007/s00586-010-1501-z

13. Chung AS, Campbell DH, Hustedt JW, Olmscheid N, Chutkan N. Inpatient outcomes in dialysis-dependent patients 
undergoing elective lumbar surgery for degenerative lumbar disease. Spine (Phila Pa 1976). 2017;42(19):1494-1501. https:// doi.org/10.1097/BRS.0000000000002122

14. Shaw KA, Boomsma SE, Johnson PC, Gloystein DM. Hemodialysis-associated spondylodiscitis of the cervical spine: a case report. JBJS Case Connect. 2015;5(4):e90. https://doi.org/ 10.2106/JBJS.CC.O.00037

15. Okada Y, Miyamoto H, Uno K, Sumi M. Clinical and radiological outcome of surgery for pyogenic and tuberculous spondylitis: comparisons of surgical techniques and disease types. J Neurosurg Spine. 2009;11(5):620-627. https://doi.org/ 10.3171/2009.5.SPINE08331

16. Lin CP, Ma HL, Wang ST, Liu CL, Yu WK, Chang MC. Surgical results of long posterior fixation with short fusion in the treatment of pyogenic spondylodiscitis of the thoracic and lumbar spine: a retrospective study. Spine (Phila Pa 1976). 2012;37(25):E1572-1579. https://doi.org/10.1097/ BRS.0b013e31827399b8

17. Kalra KP, Dhar SB, Shetty G, Dhariwal Q. Pedicle subtraction osteotomy for rigid post-tuberculous kyphosis. $J$ Bone Joint Surg Br. 2006;88(7):925-927. https://doi.org/10. 1302/0301-620X.88B7.17366

18. Yu B, Zhu K, Zhao D, Wang F, Liang Y. Treatment of extreme tuberculous kyphosis using spinal osteotomy and halo- pelvic traction: a case report. Spine (Phila Pa 1976). 2016;41(4):E237-E241. https://doi.org/10.1097/BRS.000000000 0001195

Disclosures and COI: The authors received no funding for this study and report no conflicts of interest.

Corresponding Author: Anouar Bourghli, MD, Orthopedic and Spinal Surgery Department, Kingdom Hospital, PO Box 84400, Riyadh 11671, Saudi Arabia. Phone: (966) 275-0234; Fax: (966) 275-1111; Email: anouar.bourghli@gmail.com.

Published 31 October 2019

This manuscript is generously published free of charge by ISASS, the International Society for the Advancement of Spine Surgery. Copyright (c) 2019 ISASS. To see more or order reprints or permissions, see http://ijssurgery.com. 